\title{
A RARE CASE OF HEMI-CORPUS VITILIGO
}

\author{
Kamal Kumar $\mathrm{M}^{1}$, Naveen $\mathrm{N}^{2}$
}

\section{HOW TO CITE THIS ARTICLE:}

Kamal Kumar M, Naveen N. "A Rare Case of Hemi-Corpus Vitiligo". Journal of Evolution of Medical and Dental Sciences 2015; Vol. 4, Issue 02, January 05; Page: 297-302, DOI: 10.14260/jemds/2015/46

\begin{abstract}
Vitiligo is a hypopigmentation causing malady of skin occurring in blotches. It is impossible to envisage the extent of skin that can be affected. It has no age, sex or ethnic discrimination, but most develop by the age of 20 and more noticeable in dark skin people. Some acquire sparing white dots while others have white patches affecting larger areas of the skin. It may also affect hair, the inside of the mouth and even the eyes. Most widely accepted to be of autoimmune in nature but is of multifactorial origin. It is commonly associated with other autoimmune disorders affecting thyroid, adrenals and pancreas. Many variants are described depending on the pattern and extent of the skin involved. It is clinically diagnosed sometimes requiring biopsy to differentiate from other hypopigmentation disorders. Currently available treatment options for vitiligo include sunscreens for photosensitive skin, cosmetic cover-up, restoration of normal skin color with use of medical options like PUVA or surgically by autografting, tattooing and depigmentation of normal skin to make an even color. Results of these are not constant with only partial recovery. But the most commonly neglected imperative factor in the treatment of vitiligo is the psychological trauma and fear of social disgrace sustained by the patient. In this case report, we state a rare pattern of vitiligo involving only half of the body and the importance and effect of psychological treatment.
\end{abstract}

KEYWORDS: Vitiligo, Treatment, Segmental.

INTRODUCTION: Vitiligo is a pigmentary disorder of the skin and mucous membranes that is characterized by white patches due to destruction of melanocytes. It affects around $1 \%$ of the population. Vitiligo develops early in life and half the patients first notice vitiligo before 20 years of age. It appears often in an area of minor injury, sunburn, body folds (such as armpits), areas around moles, or areas around body openings. The hypopigmented spots tend to be more noticeable on darker skin though vitiligo affects individuals of all ethnic origins and both sexes equally. Individuals with a family history of vitiligo are at increased risk and it is hereditary in one third of those affected. Vitiligo is believed to be an autoimmune disorder as people with vitiligo are also likely to have other autoimmune diseases, such as autoimmune thyroid disease (Hashimoto's thyroiditis), diabetes mellitus, alopecia areata, Addison's disease and pernicious anemia.

Vitiligo is often seen on the hands, feet or face, preferring a perioral and periocular distribution. It can also affect the eyelids and hair. Frequently rapid progressive pigment loss on several areas of the skin which are white or hypopigmented, variable in size, well demarcated; round, oval, or linear in shape, convex borders [1] and enlarge centrifugally over time at random rate. Initial appearance of these patches can be followed by a steady interval followed by cycles of pigment loss and stability.

Depending on type of bodily distribution there are three types of vitiligo - Localized (Focal, Segmental or Mucosal), Generalized (Acrofacial, Vulgaris or Mixed) and Universal (rare type). The common type is 'Vitiligo Vulgaris'. Variant varieties include linear, trichrome, quadrachrome, inflammatory and blue vitiligo. 
Generally diagnosis is made based on clinical findings. Wood's lamp is used on people with fair skin. Biopsy is seldom needed for differentiating vitiligo from other hypopigmentary disorders. Investigations to determine other associated autoimmune disorders like thyroiditis, diabetes mellitus may occasionally be called for.

Since no acknowledged treatment modality is available to cure vitiligo, numerous methods such as Cosmetics for concealment, Systemic phototherapy using UV light for re-pigmentation, Steroid therapy - systemic (prednisone) or corticosteroid creams, Depigmentation of unaffected skin parts, Micropigmentation (tattooing in dark-skinned individuals - works best for the lip area) and LASERs (Excimer laser), can be used for facade betterment of skin affected by vitiligo. Various surgical therapies are also being used such as Cultured epidermal suspensions, Thin dermoepidermal grafts, Suction epidermal grafting, Punch minigrafting and Cultured melanocyte suspensions. $[2,3]$ Medical treatments aim at immune system, and try to undo the damage. Surgical treatments are infrequently used and intend at transplant of healthy melanocytes from other areas. Both treatments may be difficult and annoyingly prolonged and hence require exceptional patient compliance. Most commonly used is treatment is PUVA (Psoralen + Ultra-Violet A light). Surgical therapy for vitiligo is considered if the patient doesn't respond to PUVA treatment even after one year of remedy.

The major predicament of vitiligo is that it is disfiguring and cause psychological ordeal to the patient. Here we present a rare variety of vitiligo involving only one side of the body.

CASE REPORT: A 35 year old unmarried male presented in plastic surgery OPD with complains of hypopigmented spots over left side of his body. These spots were distributed only on the left side and were on his trunk and limbs only. His head and neck areas were selectively spared along with the right side of the corpus. He also complained of occasional itching and pain in the hypopigmented areas. These lesions developed 10 years back and gradually intermittently attained the present pattern and have distinctly remained same since 4 years. He had been on treatment with PUVA initially for two years without any improvement in the disease progression. He was also treated with systemic steroids. Patient also had taken ayurvedic and homeopathic treatment for the same. As someone had suggested surgical treatment for his condition he presented to us inquiring for it. The patient also suffered from low esteem and depression regarding his condition and blamed it for hindrance in having a normal social life and seeking alliance for spouse. On examination, the patient had Fitzpatrick type V skin. Hypopigmented areas solitary and in clusters were noted on the trunk noticeably limited by the midline on left side of the body. Same was noted in the upper limb with lower limb being most affected. In the groin, genitalia was spared as were his head and neck region, there were hyperpigmented spots noted interspersed in hypopigmented areas [Fig. 1 \&2]. No other focal or systemic abnormalities were clinically found. Investigations included blood work up pertaining to thyroid and glucose levels which were within normal limits. Patient was counseled and explained about the surgical options including their drawbacks. Psychiatric evaluation was evident of depression and treatment for same was ensued. After 3 months patient was evaluated again. He appeared more confident with alleviated social fear and symptoms like itching had receded. Patient was advised corticosteroids cream for local application and use of sunscreen agents. Followed up for 6 months, patient is leading a better life and is not keen for surgical therapy. 
DISCUSSION: Vitiligo is a pigmentary disorder in which melanocytes are absent or non-functional resulting in hypopigmented spots. Mucosal areas of mouth, nose and the retina may be affected and the hairs on depigmented skin may also be affected (poliosis). Ancient Indian texts such as AtharvaVeda and olden Egyptian medical text Ebers Papyrus have description of the disease. Worldwide incidence is around $1 \%$ affecting as many as 65 million people,[4] with some populations averaging between $2-3 \%$ and as high as $16 \% .^{[5]}$ It affects both genders and all races equally. Vitiligo affects people of all skin types, but is more noticeable in people with darker skin.

Though cause is not entirely known researchers have several different theories including autoimmune (strongly implicated), genetic, accumulation of toxic compounds, altered cellular environment, oxidative stress, neural, or viral causes.[1] Some reports of single event triggers such as sunburn or emotional distress have been stated. Vitiligo seems to be somewhat more common in people with certain autoimmune diseases, including hyperthyroidism, adrenocortical insufficiency, alopecia areata, and pernicious anemia. Genes NLRP1, PTPN22, HLA and TYR variations have been linked with an increased risk. Inheritance pattern is complex since multiple causative factors are involved. About one-fifth of people with vitiligo have at least one affected close relative. Triggering factors suggested are stressful events such as childbirth, repetitive trauma (Koebner response) and exposure to chemicals. As understood, a combination of interrelating factors result in different degrees and rates of progression of vitiligo indicating it to be a multifactorial disease.

Prime symptom is presence of pale patchy areas of hypopigmented skin commonly seen in extremities.[1,6] Initially small, the patches often increase in size and become larger over time. The severity of vitiligo varies with the individual and is unpredictable as to pigment loss or spread rate. Other uncommon symptoms are premature hair graying, loss of colour in the retina and mucous membranes. Development of vitiligo in sites of trauma such as a cut, burn, or abrasion or pressure on the elbows, knees, and bony prominences, represent the isomorphic or 'Koebner' phenomenon. The extent and rate of color loss from vitiligo is unpredictable.

Though no physical impairment exists patients have psychological bearing such as depression, low self-esteem and emotional distress affecting their quality of life.

Types of vitiligo are localized - present in distinct sections, generalised/non-segmental (NSV) - widespread across the body (most common) and universal - near complete loss of pigment (over $80 \%$ of the body).

Vitiligo is clinically diagnosed. However, a black light (Wood's lamp) can be used in the early phase for identification especially in fair skinned individuals. Rarely biopsy may be needed to differentiate from other hypopigmentation disorders such as Lupus erythematosus , Pityriasis alba, Tuberculoid leprosy, Post inflammatory hypopigmentation, Tinea versicolor, Piebaldism, Idiopathic guttate hypomelanosis, Progressive macular hypomelanosis, Chemical leukoderma, Postinflammatory hypomelanosis and Primary adrenal insufficiency. On microscopy, there are degenerative changes in keratinocytes and melanocytes in the border lesions and adjacent skin, increased numbers of Langerhans cells, epidermal vacuolization and thickening of the basement membrane. Loss of pigment and melanocytes in the epidermis is highlighted by Fontana-Masson staining and immunohistochemistry testing. Specific investigations for associated autoimmune disorders like thyroiditis, diabetes mellitus, Addison's disease should be done if necessitates. 
Important objective of treatment is to improve the appearance of the skin. Existence of many treatment options signifies absence of universally effective and safe therapy. Factors such as extent, distribution and rate of progression of disease determine the choice of treatment.

- Sun Protection: Vitiligo is photosensitive. Sun exposure is to be minimized and ensure protective clothing and sunscreen. Hypopigmented skin should be protected from sunburn reaction and tanning of normal skin should be limited. Sunscreens with SPF of at least 30 should be used.

- Camouflage: Cosmetics can be used to cover up the patches of depigmented skin.

- Topical steroid creams - Mometasone furoate may aid in repigmentation. Also Oxsoralen (highly phototoxic) is used.

- Phototherapy: Ultraviolet (UV) radiation can be used to restore pigment to the skin. Oral and topical medications of psolarens (UV photosensitisers) - Trisoralen or Oxsoralen-Ultra combined with UVA irradiation repigment areas of skin and is moderately effective treatment with a high relapse rate and potential side effects. Narrowband ultraviolet B (NB-UVB) radiation is an effective treatment producing minimal adverse effects.

- Excimer laser Treatment: Has best results on face.

- Depigmentation Therapy: In extensive cases (>80\%), depigmentation of remaining pigmented areas of skin can be done to unify skin color and those who have failed PUVA. Topical monobenzyl ether of hydroquinone (Benoquin), more recent lasers and topical 4methoxyphenol (4-MP) can be used to remove pigment.

- Immunomodulators: Tacrolimus[7] and Pimecrolimus can be used to reduce the immune response against melanocytes.

- Calcium Modulators: Calcipotriol and Tacalcitol have been trialed as calcium transport in melanocytes of vitiligo patients is often defective.

Surgical therapies are only recommended for patients with stable vitiligo. That is, patients whose patches have not grown or spread for at least 6 months. Surgery is usually very costly and can leave scarring, hence, it is only considered after other therapies have proven ineffective.

- Autologous skin Grafts: Used for patients with small patches of vitiligo. PUVA may be required following the procedure to unify the color between the graft sites. Koebnerization in donor sites in generalized vitiligo restricts this procedure. Pebbling or cobblestone appearance of grafted site may occur.

- Skin grafts using Blisters: Blisters are created by using heat, suction, or freezing cold. The tops of the blisters are then cut out and transplanted to a depigmented skin area.

- Tattooing (Micropigmentation): manual tattooing of depigmented areas is a cheaper alternative, however is it difficult to find an exact match for skin colour. It works best for the lip area, particularly in people with dark skin.

Current research is on Afamelanotide which is in phase II and III clinical trials and promising Melanocyte transplantation. Transplantation procedures are ideal to treat segmental vitiligo which is poorly responsive to other types of treatment.

But the most important part of the treatment of vitiligo is to address psychological trauma of the patients by a mental health professional. Help to cope with vitiligo is required for patients who are stigmatized for their condition and may experience depression and similar mood disorders. Many 
treating physicians, often fail to recognize the profound social and psychological impact and is accentuated among persons with brown or black skin. The contrast between brown skin and white vitiligo spots can create a grotesque 'harlequin' appearance. In India, vitiligo/leukoderma is regarded as 'white leprosy'.[8] And thus patients can turn aggressive, feel a sense of shame, or become withdrawn and resentful. Vitiligo thus forms a major social dysfunction curtailing patients' ability to lead a normal work, social or married life. [9, 10] Restoration of self-confidence, self-esteem is important.

\section{REFERENCES:}

1. Halder, RM; Chappell, JL (2009). 'Vitiligo update'. Seminars in cutaneous medicine and surgery; 28 (2): 86-92.

2. Halder RM, et al. (2007)." 72. Vitiligo". In Wolff K, Freedberg IM, Fitzpatrick TB (eds). Fitzpatrick's dermatology in general medicine (7th ed.) New York: McGraw-Hill Professional.

3. Huggins RH, Schwartz RA, Janniger CK (2005). 'Vitiligo'. Acta Dermatovenerologica Alpina, Panonica, et Adriatica; 14 (4): 137-42.

4. Nath SK, Majumder PP, Nordlund JJ (1994)."Genetic epidemiology of vitiligo: multilocus recessivity cross-validated". American Journal of Human Genetics 55 (5): 981-90.

5. Krüger C, Schallreuter KU (October 2012)."A review of the worldwide prevalence of vitiligo in children/adolescents and adults". Int J Dermatol 51 (10): 1206-12.

6. National Institute of Arthritis and Musculoskeletal and Skin Diseases (March 2007)."What Is Vitiligo? Fast Facts: An Easy-to-Read Series of Publications for the Public Additional".

7. Tjioe, M; Vissers, WH; Gerritsen, MJ (2006)."Topical macrolide immunomodulators: a role in the treatment of vitiligo?" American journal of clinical dermatology 7 (1): 7-12.

8. Fitzpatriack TB, Eisen AZ, Wolff K, etal.”Disorders of Pigmentation" In: Dermatology In General Medicine, 4th ed., edited by TB Fitzpatrick et al. New York, McGraw-Hill, 1993.

9. Fitzpatrick TB, Johnson RA, Woff K etal. "Vitiligo" In: Color Atlas and Synopsis of Clinical Dermatology, $3^{\text {rd }}$ ed. New York, McGraw-Hill, 1997.

10. Ortonne JP. Mosher DB. Fitzpatrick TB. Vitiligo and Other Hypomelanoses of Hair and Skin. New York, Plenum Publishing Corporation, 1983.

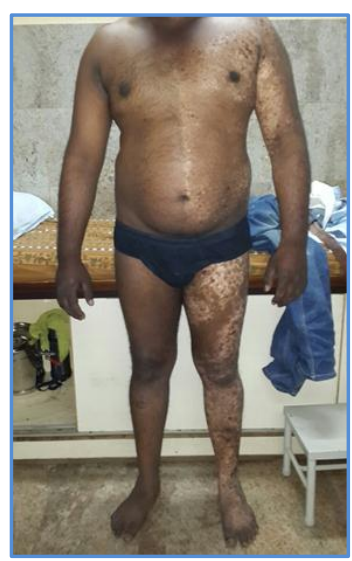

Fig. 1: Front view - note the patches limited

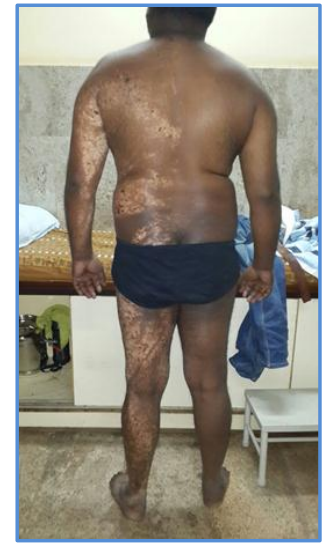

Fig. 2: Back view by midline on the trunk 


\section{AUTHORS:}

1. Kamal Kumar M.

2. Naveen $\mathrm{N}$.

\section{PARTICULARS OF CONTRIBUTORS:}

1. Associate Professor, Department of Plastic Surgery, Raja Rajeswari Medical College \& Hospital, Bangalore.

2. Senior Resident, Department of Plastic Surgery, Raja Rajeswari Medical College \& Hospital, Bangalore.

\section{NAME ADDRESS EMAIL ID OF THE} CORRESPONDING AUTHOR:

Dr. Naveen $\mathrm{N}$, \# 90, 14 ${ }^{\text {th }}$ Main, $14^{\text {th }}$ Cross, $2^{\text {nd }}$ Phase, West of Chord Road, Mahalakshmi Puram, Bangalore-560086.

E-mail: naveen_uno1@yahoo.co.in

Date of Submission: 19/12/2014.

Date of Peer Review: 20/12/2014. Date of Acceptance: 29/12/2014. Date of Publishing: 05/01/2015. 